International perspective

\title{
The Road To Rio: Medical and Scientific Perspectives on the 2016 Paralympic Games
}

\author{
Authors: \\ Cheri A. Blauwet, MD ${ }^{1,2}$ \\ Jan Lexell, MD, $\mathrm{PhD}{ }^{1,3}$ \\ Wayne Derman, MD, PhD ${ }^{1,4,5}$ \\ Guzel Idrisova, MD ${ }^{1,6}$ \\ James Kissick, MD ${ }^{1,7}$ \\ Jaap Stomphorst, MD ${ }^{1,8}$ \\ Yetsa Tuakli Wosornu, MD ${ }^{1.2}$ \\ Peter Van de Vliet, PhD ${ }^{1,9}$ \\ Nick Webborn, MD ${ }^{1,10}$
}

\author{
Affiliations: \\ ${ }^{1}$ Medical Committee, International Paralympic Committee, Bonn, Germany \\ ${ }^{2}$ Department of Physical Medicine and Rehabilitation, Spaulding Rehabilitation Hospital and Brigham and \\ Women's Hospital, Harvard Medical School, Boston, USA \\ ${ }^{3}$ Department of Health Sciences, Rehabilitation Medicine Research Group, Lund University, Lund, \\ Sweden; Department of Neurology and Rehabilitation Medicine, Skåne University Hospital, Lund, \\ Sweden; Department of Health Science, Luleå University of Technology, Luleå, Sweden \\ ${ }^{4}$ Institute for Sport and Exercise Medicine, Division of Orthopaedics, Stellenbosch University, South Africa \\ ${ }^{5}$ International Olympic Committee (IOC) Research Centre, South Africa \\ ${ }^{6}$ Lesgaft National State University of Physical Education, Sport and Health, St. Petersburg, Russia; \\ National Paralympic Committee of Russia, Moscow, Russia \\ ${ }^{7}$ Department of Family Medicine, University of Ottawa, Ottawa, Canada; Carleton University Sport \\ Medicine Clinic, Ottawa, Canada \\ ${ }^{8}$ Sports Medicine Department, Isala Klinieken, Zwolle, The Netherlands \\ ${ }^{9}$ Medical and Scientific Department, International Paralympic Committee, Bonn, Germany \\ ${ }^{10}$ School of Sport and Service Management, University of Brighton, East Sussex, United Kingdom
}

\section{Corresponding Author:}

Cheri A. Blauwet, MD

Spaulding Rehabilitation Hospital and the Brigham and Women's Hospital

Department of Physical Medicine and Rehabilitation, Harvard Medical School 
300 First Avenue

Charlestown, MA, USA

Email: cblauwet@partners.org

Office: +01617 7329712

Fax: +01617 7329576 
In August and September of this year, the world will turn its attention to Rio de Janiero, Brazil, for the 2016 Summer Olympic and Paralympic Games. Of interest to physiatrists, the Paralympic Games will take place from September 7-18, with an estimated total of 4 billion viewers. In the United States, for the first time in history, the Summer Games will be broadcast over a total of 66 hours on NBCUniversal. The Paralympic Games represent the pinnacle of elite sport for athletes with disabilities, while also changing perceptions around the importance of grassroots sport and physical activity opportunities for the disability community more broadly.

It is no secret that the planning and preparation for the Rio Games has brought with it a number of challenges - the Zika virus, water quality, construction delays and the oft tenuous state of the Brazilian political system, to name a few. In some respects, these challenges are important as they stimulate discourse about the future of the Olympic and Paralympic Movement. In Paralympic sport, some of our current and most salient challenges are rooted in principles of sports medicine and science, as outlined below. Since it is imperative to uphold the highest standards of athlete health and safety at the Games, this presents an unparalleled opportunity for the voice of physiatrists to come to the fore. As experts in disability and functional performance, neurologic and musculoskeletal rehabilitation, and sports medicine, we are uniquely suited to make an important and timely impact on Paralympic sport. Here we provide a snapshot of what to watch out for in Rio.

\section{Prosthetic Use in Amputee Track and Field}

The use of cutting-edge prosthetics in amputee sport initially gained global attention through the story of Oscar Pistorius - the first amputee to compete in both the Olympic and Paralympic Games in London, 2012. Since that time, amputee athletes have sought to compete in both disability-specific and ablebodied sport, transcending stereotypes and perceived societal limitations. Most recently, a German long jumper and single below knee amputee, Markus Rehm, has garnered international notoriety. At the 2015 Doha International Paralympic Committee (IPC) Athletics World Championships, Rehm jumped a length of 8.40 meters, making him the new IPC world record holder [1]. Lesser known, however, is that this same jump would have made him a gold medalist at the London 2012 Olympic Games. In his attempt to compete in able-bodied track and field, Rehm recently underwent biomechanics testing at the German Sport University of Cologne. The results of the testing were inconclusive on whether he has an advantage over his able-bodied counterparts. Given that the International Association of Athletics Federations (IAAF) rules require him to prove that he has no advantage, he remains barred from able-bodied competition until further evidence is brought to the fore [2]. Looking ahead, it is clear that Paralympic athletes will continue to challenge concepts of what constitutes an appropriate, and fair, use of technology, versus unfair performance enhancement. As with all cutting edge technologies, this pushes us out of our comfort zone and begs the question - what will the future bring? 


\section{Anti-Doping Challenges}

Prior to the Olympic and Paralympic Games, doping in sport inevitably gains attention as athletes push the envelope to enhance performance, sometimes through the use of prohibited substances or methods. In Paralympic sport, issues related to an athlete's disability may, at times, lead to unique and complex challenges related to sample collection, the interpretation of results, and the granting of therapeutic use exemptions (TUEs) that are central to conducting a rigorous testing program. For example, in 2014 a paper was published noting that several Japanese athletes had tested positive for glycerol - a substance that is prohibited given its potential for use as a masking agent [3]. Upon further investigation, it was discovered that these positive tests were due to the athletes' use of a glycerol-containing lubricant for self-catheterization [3]. Shortly thereafter, the World Anti-Doping Agency (WADA) testing standards were adjusted to increase the urine threshold value that constitutes a positive test, making this error unlikely to occur in the future. In the lead-up to Rio, all athletes have been cautioned against the use of glycerolcontaining lubricants for urine sample collection. Additionally, the Rio Paralympic village polyclinic and venue medical stations, as well as all doping control stations, will stock only lubricants that do not contain glycerol. As the performances of elite Olympians and Paralympains remain under scrutiny from an antidoping perspective, considerations such as this, which uniquely impact athletes with a disability, must be taken into account to ensure fair and equitable competition.

\section{Use of Botulinum Toxin and Potential Impact on Classification}

For physiatrists, it is commonly known that botulinum toxin is a standard treatment for spasticity caused by central neurologic injury such as cerebral palsy, traumatic brain injury, or stroke. Botox blocks the release of acetylcholine at the neuromuscular junction, thereby inhibiting the reflex muscle contraction that is the hallmark of spasticity [4]. From a functional standpoint, in appropriate candidates, therapeutic botulinum toxin may enable increased range of motion and reduce painful spasticity, however with a trade-off of causing motor weakness. Additionally, from a global perspective, botulinum toxin is often seen as a "luxury" treatment that is readily available only within higher-resourced health care systems. In Paralympic sport, concern has been raised regarding the use of botulinum toxin by athletes in order to unfairly improve performance. Currently, little objective evidence exists to justify this claim, although it has prompted concern from athletes and coaches, particularly those from resource-limited settings. For example, a wheelchair racer with cerebral palsy who has not yet undergone treatment with botulinum toxin may be classified as a T33, characterized by mild upper extremity spasticity and resultant asymmetry in propulsion. Shortly thereafter, he/she maybe undergo treatment, and in follow-up, present with the function of an athlete in class T34, characterized by minimal upper extremity spasticity and symmetry in propulsion. Should this athlete be re-classified post-botulinum toxin injection? Under the current regulations in some sports, an athlete is to inform the Head of Classification of any medical intervention that may affect his or her impairment, in which case the athlete is requested to present for reclassification. However, there is a need for further standardization. Additionally, as botulinum toxin is 
currently not a substance on the World Anti-Doping Agency Prohibited List, its use cannot be objectively monitored through the therapeutic use exemption (TUE) process. As medical and scientific leaders in Paralympic sport, we must be cognizant of these challenges and strive to uphold the rights of all athletes, including those from under-resourced regions throughout the Movement [5].

\section{Concussion and its Impact on Paralympians}

Concussion is a very important topic in sports medicine, with increasing resources being committed to the expansion of research, clinical care, and educational initiatives to potentially reduce its incidence and to provide better treatment paradigms. The next major update on concussion in sports science is expected at the $5^{\text {th }}$ International Consensus Conference on Concussion in Sport, due to take place in Berlin in October 2016. Within this context and from a historical perspective, little attention has been placed on the unique needs of Paralympic athletes, for whom the risk of concussion in sport is of equal concern.

Several categories of Paralympic sport, for example, those uniquely developed for athletes with visual impairment (e.g. football 5-a-side) and those that take place a high speeds (e.g. Para alpine sit skiing) show a particularly high incidence of concussion [6,7]. Additionally, large portions of the SCAT3 (Sport Concussion Assessment Tool $3^{\text {rd }}$ Edition) are not useful for athletes who use wheelchairs or with baseline balance deficits, and little is known regarding how the effect of concussion may be unique in athletes with central neurologic injury such as cerebral palsy, stroke, or traumatic brain injury. At the Rio 2016 Paralympic Games, the IPC Injury and IIIness Surveillance Study will again be conducted to provide baseline epidemiologic data regarding injuries and illnesses in Paralympic sport, with a goal to reduce their incidence [8]. This coming iteration of the study will be expanded to include additional details regarding concussion, thus providing novel data regarding risk factors at the Paralympic Games. Given the growth of Paralympic sport participants, further longitudinal, prospective studies are urgently needed to discern the unique needs of athletes with disabilities related to the prevention, incidence, diagnosis, and treatment of concussion.

\section{Autonomic Dysreflexia and Boosting}

For decades, the practice of "boosting" has been under scrutiny in Paralympic sport. Boosting is the intentional induction of autonomic dysreflexia for unfair performance enhancement by athletes with high thoracic and cervical spinal cord injury, and it is strictly prohibited by the IPC due to its inherent health risks. Since 2008, the IPC has implemented a testing program for boosting in major Paralympic competition, with the goal of deterring its practice [9]. Historically, systolic blood pressure (SBP) was utilized as a proxy marker for boosting. Athletes were tested just prior to competition, and a SBP reading of $\geq 180 \mathrm{mmHg}$ was considered a "positive" test. Over time, the strengths and limitations of this testing paradigm have been evaluated. From the years 2008-2015, a total of 159 tests were conducted, none of which were positive. Additionally, it was noted that athletes could be dysreflexic with SBP levels much less than $180 \mathrm{mmHg}$. Utilizing historic data of pre-competition baseline SBPs in athletes with spinal cord 
injury, and supported by recent research findings [10], the IPC Governing Board (upon recommendation of the IPC Medical Committee) has recently amended its rules to lower this threshold for a "positive" test to $>160 \mathrm{mmHg}$, effective immediately and to be implemented in Rio [8]. The primary goal of this program is to deter athletes from engaging in the practice of boosting given its inherent dangers, and to raise awareness regarding the importance of self-monitoring amongst athletes who may be susceptible to autonomic dysreflexia [11].

\section{Conclusion}

The Rio Paralympics will undoubtedly present exciting and cutting-edge performances from a diverse spectrum of athletes with disabilities. Competing and working within an environment such as Rio is likely to add complexity to issues such as infection control, accessibility, and safety. Although it is difficult to estimate the magnitude of the impact of these challenges, it is clear that in some respects athletes with disabilities may be disproportionately impacted. The same will likely be said for the Paralympic Games in Pyeongchang in 2018, or Tokyo in 2020; each Paralympic Games will present unique challenges, and opportunities, for both athletes and team physicians.

Although the topics outlined in this article will push our limits in providing optimal care to Paralympians, historically, the lessons learned from past Paralympic games have translated into future advances in sports medicine and science, benefiting athletes with disabilities across a spectrum of athletic ability. Perhaps, in the next 2 or 3 Paralympic games, athletes will be utilizing sports prostheses made from a 3D printer, or an investigator will develop a specialized sideline assessment for concussion in athletes with dyskinetic cerebral palsy. As sports physicians, our charge is to innovate and to develop evidence that promotes athletes' health and performance. Further, as the physiatric members of the team of physicians, our collective voice is essential to maintain athletes' health and function in the presence of a wide range of disabilities. Paralympic athletes are watching. Indeed, the entire world will be watching!

\section{References}

1. Results of the 2016 International Paralympic Committee Athletics World Championships. Accessed July 1, 2016 at https://www.paralympic.org/doha-2015/schedule-results.

2. Potthast $\mathrm{W}$, Hobara $\mathrm{H}$, Gabrowski $A$. Biomechanical comparison of the long jump of athletes with and without a below the knee amputation. Report presented at Press Conference Markus Rehm, 30 May 2016, Cologne, Germany.

3. Okana M, Nishitani Y, Kageyama S. Possibility of analytical finding of glycerol caused by selfcatheterization in doping control. Drug Test Anal 2014;6:1151-4.

4. Kumar R, Dhaliwal HP, Kukreja RV, Singh BR. The Botulinum Toxin as a therapeutic agnet: molecular structure and mechanism of action in motor and sensory systems. Semin Neurol 2016;36:10-9. 
5. McNamee M, Savulescu J. Ethical considerations in Paralympic Sport: When are elective treatments allowable to improve sports performance? PM R 2014;6(8 Suppl):S66-76.

6. Willick SE, Webborn N, Emery C, Blauwet CA, Pit-Grosheide P, Stomphorst J, Van de Vliet P, Patino Marques NA, Martinez-Ferrer JO, Jordaan E, Derman W, Schwellnus M. The epidemiology of injuries at the London 2012 Paralympic Games. Br J Sports Med 2013;47:426432.

7. Derman W, Schwellnus MP, Jordaan E, Runciman P, Van de Vliet P, Blauwet C, Webborn N, Willick S, Stomphorst J. High incidence of injury at the Sochi 2014 Winter Paralympic Games: a prospective cohort study of 6564 athlete days. Br J Sports Med. 2016 (DOI 10.1136).

8. Derman W, Schwellnus M, Jordaan E, Blauwet CA, Emery C, Pit-Grosheide P, Marques NA, Martinez-Ferrer O, Stomphorst J, Van de Vliet P, Webborn N, Willick SE. Illness and injury in athletes during the competition period at the London 2012 Paralympic Games - development and implementation of a web-based surveillance system (WEB-IISS) for team medical staff. $\mathrm{Br} \mathrm{J}$ Sports Med 2013;47:420-425.

9. Blauwet $\mathrm{CA}$, Benjamin-Laing $\mathrm{H}$, Stomphorst J, Willick $\mathrm{S}$. Testing for boosting at the Paralympic Games: policies, results, and future directions. Br J Sports Med 2013;47:832-837.

10. Gee CM, West CR, Krassioukov AV. Boosting in elite athletes with spinal cord injury: A critical review of physiology and testing procedures. Sports Med 2015;45:1133-1142.

11. Bhambhani Y, Mactavish J, Warren S, Thompson WR, Webborn A, Bressan E, De Mello MT, Tweedy S, Malone L, Frojd K, Van De Vliet P, Vanlandewijck Y. Boosting in athletes with highlevel spinal cord injury: knowledge, incidence and attitudes of athletes in paralympic sport. Disabil Rehabil 2010;32:2172-90. 